\title{
APLICAÇÃO DE TÉCNICAS DE ANÁLISE SÍNCROTRON EM ARQUEOLOGIA
}

APPLICATION OF SYNCHROTRON ANALYTICAL TECHNIQUES IN ARCHEOLOGY

Douglas Galante

Fabio Rodrigues

Lara Maldanis

Tamires Gallo

Como citar este artigo:

GALANTE, Douglas; RODRIGUES, Fabio; MALDANIS, Lara; GALLO, Tamires. Aplicação de técnicas de análise Síncotron em Arqueologia. In: Cadernos do Lepaarq, v. XV, n.30., p. 277-289, Jul-Dez. 2018. 


\section{Aplicação de técnicas de análise Síncotron em Arqueologia}

Douglas Galante

Fabio Rodrigues ${ }^{\mathrm{b}}$

Lara Maldanis ${ }^{\mathrm{a}, \mathrm{c}}$

Tamires Gallo

\begin{abstract}
Resumo: Este artigo apresenta uma revisão sobre os principais métodos analíticos atualmente empregados no estudo de bens culturais e arqueológicos, com especial foco em técnicas baseadas em síncrotron, explorando suas vantagens e limitações dentro das demandas desse campo. As técnicas abordadas são a tomografia de raios X para análises de imagem do interior de objetos, a fluorescência de raios $\mathrm{X}$ para o mapeamento químico da superfície dos objetos de interesse e a absorção de raios X para a especiação química de determinados elementos. Tais técnicas encontram-se disponíveis na fonte de luz síncrotron brasileira do Laboratório Nacional de Luz Síncrotron, o qual atualmente constrói Sirius, uma fonte de 4a geração que irá levar o Brasil a novas fronteiras tecnológicas para estudos de materiais, tal qual na área de arqueometria.
\end{abstract}

\section{Palavras Chave:}

Arqueometria; Síncrotron; Arqueologia; Micro-tomografia. Espectroscopia.

\begin{abstract}
This article presents a review about the main analytical methods currently applied in the study of cultural heritage and archeology, with special focus on synchrotron-based techniques, exploring the advantages and limitations within the requirements of the field. The techniques presented are X-ray tomography for imaging the interior of objects, $\mathrm{X}$ - ray fluorescence for chemical mapping of the surface of objects of interest and X-ray absorption for chemical speciation of elements. These techniques are available in the Brazilian synchrotron light source of the Brazilian Synchrotron Light Laboratory, which currently builds Sirius, a 4th generation source that will take Brazil to new technological frontiers for the study of materials, as in the field of archaeometry.
\end{abstract}

\section{Keywords:}

Synchrotron; Archaeometry; Archeology; Micro-CT;

Spectroscopy.

$\boldsymbol{a}$ Laboratório Nacional de Luz Síncrotron (LNLS), Centro Nacional de Pesquisa em Energia e Materiais (CNPEM), CEP 13083-970, Campinas, São Paulo, Brasil. E-mail: douglas.galante@lnls.br b Departamento de Química Fundamental, Instituto de Química (IQ), Universidade de São Paulo (USP), CEP 05513-979, São Paulo, São Paulo, Brasil. $c$ Instituto de Física de São Carlos (IFSQ), Universidade de São Pauto (USP), CEP 13566-590, São Carlos, São Paulo, Brasil. 


\section{INTRODUÇÃO}

A aplicação de técnicas analíticas em arqueologia, conhecida como arqueometria, vem sendo desenvolvida e empregada em todo o mundo desde os anos 1960; no Brasil, diversas pesquisas nessa área também têm sido reportadas (Appoloni 2013). Essa abordagem vem ganhando aceitação e adoção pela comunidade arqueológica como uma forma de refinar e detalhar o conhecimento sobre artefatos e sítios arqueológicos em diferentes escalas, da macroscópica à nanométrica. É sempre importante ressaltar que, apesar da arqueometria poder fornecer importantes informações sobre os elementos materiais, ela não deve ser feita de forma desconexa dos elementos imateriais, culturais e históricos. Se feita dessa forma, os dados coletados, por mais sofisticados que sejam, em nada contribuiriam para trazer à luz a forma passada de ser e viver humano. Dessa maneira, o profissional realizando pesquisa em arqueometria, muitas vezes um químico ou físico, deve manter-se em constante contato e interação com cientistas das áreas de humanidades, de maneira a criar um corpo coeso de informações sobre os elementos que estuda.

Este breve resumo sobre técnicas analíticas aplicadas à arqueometria não tem a pretensão de abordar toda a dimensão multi e interdisciplinar da área, por sua grande complexidade. Ao invés, propõe-se que dialogue com os outros textos desse conjunto, brevemente apresentando algumas das ferramentas analíticas disponíveis aos arqueólogos. Nessa área, podemos evidenciar duas abordagens usadas pela comunidade: 1) Medidas em campo, com equipamentos portáteis; 2) Medidas em laboratório, com equipamentos mais sofisticados e sensíveis. Apesar de ser tentador escolher uma em detrimento da outra, as duas são importantes e complementares. As técnicas de campo permitem medidas in situ, garantindo mínima alteração a obras que podem ser irremovíveis, porém também são mais limitadas em sua sensibilidade, resolução e em tipo de técnica disponível. As técnicas de laboratório podem ser extremamente mais sensíveis, com altíssima resolução espacial e espectral. No entanto, obviamente requerem o transporte do material ou a coleta de amostras do mesmo, o que pode ser impossível em muitos casos. Apesar de técnicas de campo serem de extrema importância para a arqueologia, elas ultrapassam o escopo deste texto, portanto nos restringiremos às técnicas de laboratório. As técnicas físicas e químicas usadas em arqueometria são as mesmas empregadas em outras áreas, como ciência dos materiais, porém com algumas restrições especiais devido ao tipo de amostra:

Preservação do material original: grande parte das técnicas de análise físicas e químicas é destrutiva, o que não é aceitável para tratar materiais com valor histórico, cultural, artístico ou mesmo econômico. Dessa maneira, é fundamental que a arqueometria se baseie em técnicas e metodologias minimamente destrutivas e/ou invasivas para a amostra, e que o efeito de seus usos em diferentes tipos de materiais seja bem conhecido. Para isso, a comunidade científica vem se organizando para criar um corpo de conhecimento sobre como mitigar os danos em bens culturais. Por exemplo, a IAEA - International Atomic Energy Agency - organiza encontros técnicos regulares sobre o tema, com os maiores especialistas da área, das ciências humanas e exatas.

Portabilidade: como os materiais arqueológicos frequentemente estão em campo, em sítios arqueológicos, ou depositados em coleções de museus, a remoção dos mesmos sem danos em transporte para laboratório, pode ser muito complexa, dispendiosa ou mesmo impossível. Nesse caso, o desenvolvimento de equipamentos portáteis é fundamental, e essa área tem se beneficiado com o rápido desenvolvimento da eletrônica e informática das últimas décadas. Hoje há equipamentos para análise rápida e de alta qualidade, facilmente transportáveis. No entanto, a análise com equipamentos portáteis será sempre mais limitada que em equipamentos em laboratório, e o custo-benefício deve ser avaliado para cada caso. 
Minimo gasto de amostra: quando é impossível evitar os danos ou fazer as medidas in situ, é desejável que a técnica utilize o mínimo possível de material da amostra. Isso tem sido possível atualmente com o desenvolvimento de técnicas cada vez mais sensíveis, permitindo a análise com quantidades diminutas, da ordem de microgramas ou em escalas nanométricas. Nesse quesito, as técnicas síncrotron, que serão apresentadas a seguir, mostram-se excepcionais, permitindo a análise de quantidades muito pequenas de materiais. A tendência é que esse desenvolvimento continue para permitir o menor dano possível nos objetos em estudo.

Devido ao desconhecimento das técnicas e ao custo relativamente alto dos equipamentos, especialmente para a realidade brasileira, a arqueometria ainda é uma abordagem usada apenas por alguns grupos de pesquisa, mas está em franca expansão. Atualmente já existem diversos laboratórios no país capazes de realizar análises com materiais arqueológicos sem custos para os pesquisadores, e que, em muitos casos, estão ainda sendo subutilizados por essa comunidade específica. Como exemplo, podemos citar o Laboratório Nacional de Luz Síncrotron (LNLS) ${ }^{1}$ e o Laboratório Nacional de Nanotecnologia (LNNano $)^{2}$, ambos parte do Centro Nacional de Pesquisa em Energia e Materiais (CNPEM ${ }^{3}$, Campinas-SP). Os laboratórios possuem instalações de pesquisa de última geração abertas à toda comunidade acadêmica gratuitamente, sendo essa sua principal missão. O acesso é feito via submissão de projetos, que são analisados por seu mérito de forma competitiva, de maneira a utilizar o tempo disponível nos equipamentos da forma mais produtiva. O CNPEM conta ainda com um programa de financiamento próprio para custear usuários com projetos selecionados que sejam de fora do estado de São Paulo, em uma tentativa de minimizar a distribuição desigual de recursos humanos e tecnológicos existentes no país. O LNLS/CNPEM construiu o primeiro síncrotron do hemisfério sul, estando em operação ininterrupta desde 1997. Ele é um laboratório de referência no país e na região, atendendo principalmente pesquisadores da América Latina, mas também de outras regiões.

Nas instalações de pesquisa do laboratório é possível realizar variadas técnicas de caracterização e microscopia eletrônica, de raios X, infravermelho, Raman, entre outras, contando com uma equipe de técnicos e pesquisadores treinados para dar suporte aos usuários externos. Além do CNPEM, há outros centros instalados em diferentes universidades do país, com equipamentos multiusuários, e mesmo uma rede colaborativa no tema (LAPAC - Rede de Laboratórios com Aplicações em Patrimônio Cultural do Brasil $)^{4}$. Além dos laboratórios e equipamentos adequados para as análises arqueométricas, é importante ainda um maior investimento na formação de alunos e pesquisadores aptos a transitar entre a técnica e os problemas científicos das áreas correlatas à arqueologia.

\section{TÉCNICAS BASEADAS EM SÍNCROTRON}

Um acelerador síncrotron, como o existente no Laboratório Nacional de Luz Síncrotron (LNLS/CNPEM), é um equipamento de grande porte (o acelerador do LNLS ocupa um prédio de $2500 \mathrm{~m}^{2}$ ) que gera luz a partir da aceleração de elétrons. A luz gerada tem propriedades especiais, como a grande faixa coberta do espectro eletromagnético (do infravermelho até os raios X) e o altíssimo brilho, tipicamente 1 milhão de vezes maior que equipamentos convencionais de laboratório, sejam lâmpadas ou tubos de raios X. Uma vez gerada, a luz é direcionada para diferentes estações experimentais, onde são realizadas as medidas (Figura 1). Cada linha de luz do laboratório é dedicada a uma faixa de energia diferente (Figura 2), ou

\footnotetext{
$1 \quad$ http://www.lnls.cnpem.br

2 http://www.Innano.cnpem.br

3 http://www.cnpem.br

4 http://www.dfn.if.usp.br/lapac
} 
a um tipo diferente de técnica de medida (sendo as principais as de imageamento, fluorescência, espectroscopia, difração e espalhamento).

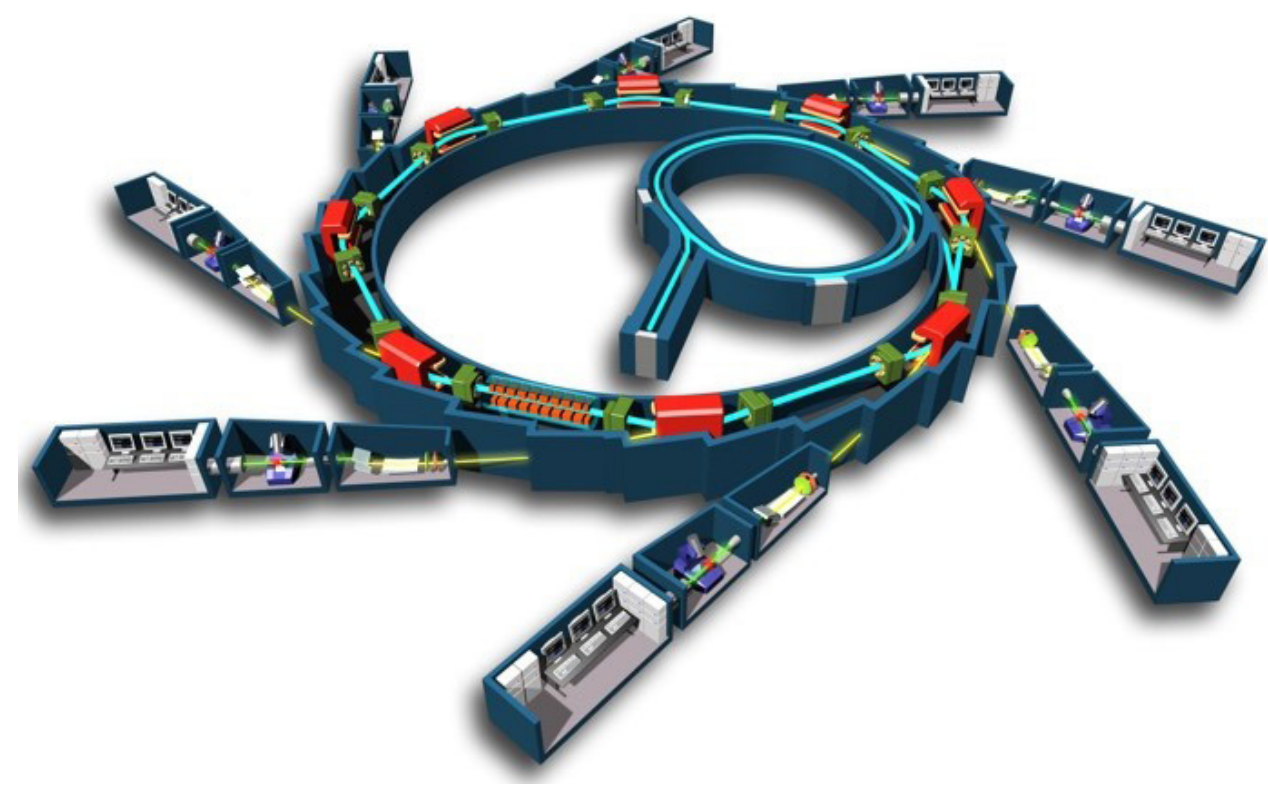

Figura 1: Esquema de uma fonte síncrotron, com o anel de armazenamento de elétrons e as linhas de luz posicionadas tangencialmente a ele, onde são realizados os experimentos. Fonte: https://fr.wikipedia.org/wiki/SOLEIL.

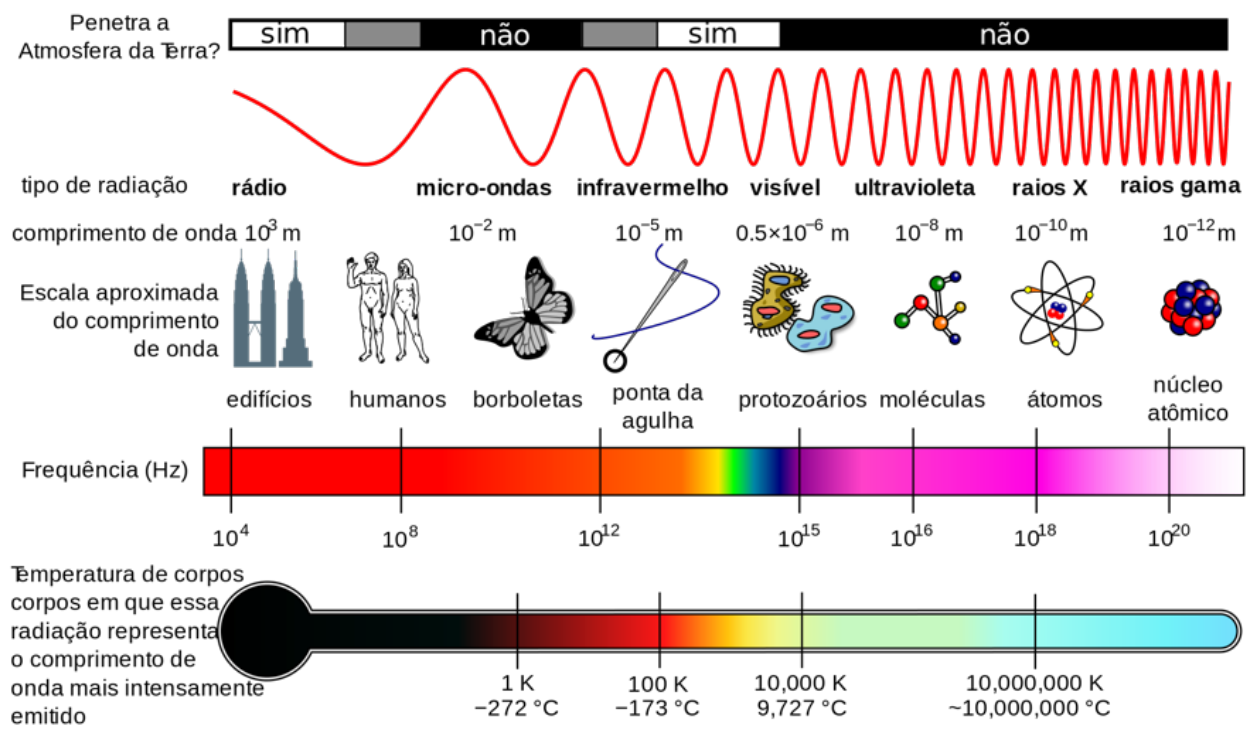

Figura 2: Espectro eletromagnético, com as escalas de tamanho e objetos que podem ser estudadas com cada tipo de radiação. Fonte: Khemis https://commons.wikimedia.org/w/index.php?curid=41541569.

Essas características únicas permitem a aplicação de uma grande diversidade de técnicas de análise que só podem ser realizadas em síncrotron. A versatilidade de cobrir uma grande faixa espectral permite que diversas técnicas possam ser aplicadas. Em baixa energia, no infravermelho, visível ou ultravioleta, é possível medir a composição e a química em nível molecular. Em energias mais altas, na faixa de raios X, é possível medir a composição atômica e elementar das amostras, além de estudar sua morfologia bi e tridimensional, sua estrutura mineral e cristalina, sendo usada como poderosa e versátil ferramenta em arqueometria (Uda, Demortier, and Nakai 2005). Algumas das técnicas foram selecionadas por sua aplicabilidade direta em arqueometria (Bertrand et al. 2012; Janssens 2011), e serão brevemente apresentadas a seguir. 


\section{Análise por imagem: tomografia computadorizada}

A técnica de tomografia, amplamente conhecida como tomografia computadorizada ou CT, da sigla em inglês, é uma ferramenta importante para o imageamento do interior de objetos em 3D de forma não-destrutiva (ou minimamente destrutiva) e não-invasiva. Ela consiste, basicamente, em recuperar a informação do volume da amostra a partir de radiografias obtidas em diferentes ângulos, utilizando-se de processamento computacional através de algoritmos de reconstrução. As radiografias, por si só, já são uma importante forma de acesso ao interior dos objetos, contendo toda a informação projetada em uma única imagem. Entretanto, quando se deseja obter informação em profundidade, para avaliar a distribuição espacial dos elementos no interior de uma amostra, faz-se necessária a aplicação da técnica de tomografia.

O tipo de informação que será obtida por tomografia dependerá da radiação utilizada e também do tipo de interação física observada. A radiação incidida em determinado material irá interagir ao longo de seu trajeto no interior do mesmo e terá sua intensidade reduzida, principalmente devido ao fenômeno de absorção. Essa radiação será atenuada dependendo das características do material, como composição química, densidade e estrutura, e também da própria natureza da radiação. A principal faixa de energia usada nas medidas é a de raios $\mathrm{X}$, como usado nas fontes síncrotron, e nêutrons, em reatores nucleares, como no IPEN (Instituto de Pesquisas Energéticas e Nucleares - São Paulo, SP). As duas modalidades fornecem informações complementares devido às suas diferenças em atenuação. Os raios X têm sua absorção diretamente relacionada com o número atômico $(Z)$, de forma que atravessam facilmente materiais leves e são altamente absorvidos por materiais densos, como metais e rochas. Já os nêutrons atravessam com grande facilidade os elementos pesados, e são mais absorvidos por elementos leves, como hidrogênio, carbono e oxigênio.

A tomografia baseada na absorção dos raios X é a técnica de CT mais comumente aplicada em estudos de arqueometria, por se tratar de uma técnica bastante difundida (em especial devido à sua vasta aplicação na medicina), e também bastante acessível, graças à disponibilidade e variedade de tomógrafos de laboratório, de diversos tamanhos e que atingem diferentes resoluções espaciais. A informação obtida por essa técnica é basicamente relacionada às diferenças de densidade ou número atômico dos materiais que compõem a amostra. Ela já é amplamente utilizada no estudo de fósseis, permitindo análises morfológicas, reconstruções anatômicas e acesso a estruturas internas preservadas nos remascentes dos organismos. A tomografia por absorção de raios $\mathrm{X}$ também teve seu potencial demonstrado para a leitura de rolos de pergaminho sem a necessidade de desenrolá-los (Baum et al. 2017), baseando-se no fato de que as tintas utilizadas nesses materiais usualmente são à base de ferro, gerando grande diferença de densidade em relação à estrutura de fibras vegetais ou animais (Mills et al. 2012). O uso desse tipo de abordagem permite a análise de artefatos e bens culturais com uma grande redução de seu manuseio, contribuindo para sua preservação (Figura 3). Entretanto, muitos materiais não apresentam uma diferença de densidade ou número atômico suficientes para gerar contraste por absorção. Nesse caso, pode-se explorar outro mecanismo de interação dos raios X com a matéria, como a refração, utilizada na técnica chamada tomografia de contraste de fase. Esse tipo de informação permite um realce nas interfaces dos diferentes materiais, contribuindo para a geração de contraste em componentes mais leves ou de densidade homogênea. Uma das formas mais simples de se realizar a tomografia de contraste de fase é através da propagação do feixe transmitido (após passar pela amostra), o que só é possível ser feito em fontes de raios X chamadas coerentes, ou seja, que produzem feixes com ondas em fase, como é o caso das fontes síncrotron.

$\mathrm{O}$ uso de fontes de raios $\mathrm{X}$ do tipo síncrotron possibilitou um grande avanço em estudos de arqueometria. Além de serem importantes para a obtenção de contraste de fase, tais fontes produzem radiação ordens de magnitude mais intensas que as fontes de tomógrafos de laboratório, permitindo a obtenção de imagens em um menor tempo de aquisição, e atingindo resoluções espaciais da ordem de micrômetros, ou até dezenas de nanômetros, em alguns casos. A utilização dessa técnica 
permitiu a identificação de tecidos moles preservados no interior de fósseis (Maldanis et al. 2016), a visualização de detalhes nas linhas de crescimento de esmalte e dentina em dentes de hominídeos (Smith et al. 2015), e foi utilizada também para a análise de rolos de papiro altamente danificados, como os mais antigos fragmentos do Velho Testamento encontrados em En-Gedi (Figura 3) (Seales et al. 2016) e os papiros carbonizados pela erupção do Vesúvio (Mocella et al. 2014), onde alguns caracteres puderam ser identificados mesmo após a carbonização, em rolos já impossibilitados de serem desenrolados. O Brasil conta com uma linha de microtomografia de raios X (linha IMX) no LNLS/CNPEM, permitindo medidas com resolução sub-micrométrica.

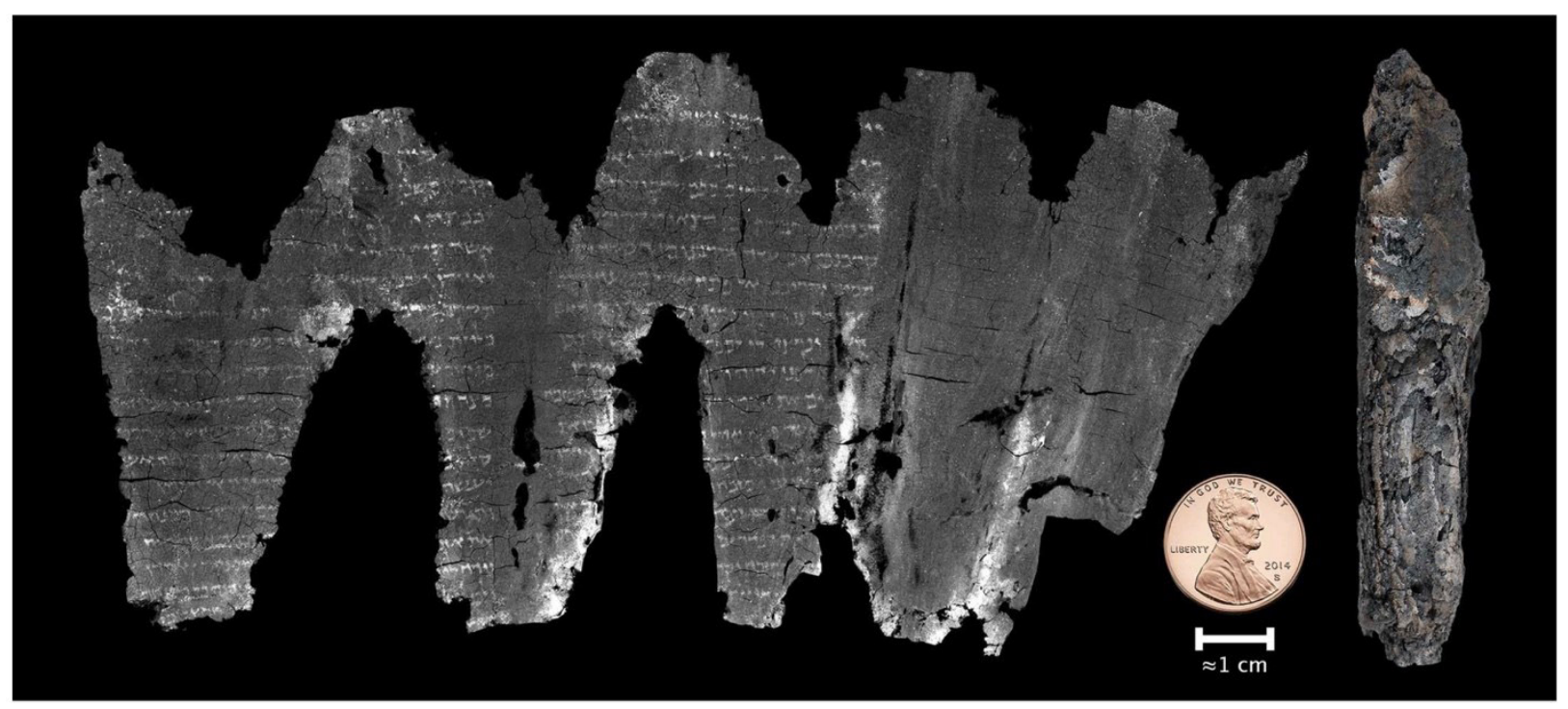

Figura 3: Papiro de En-Gedi, a mais antiga cópia do Velho Testamento, desenrolada virtualmente por micro-CT. Fonte:(Seales et al. 2016) ${ }^{5}$

A principal desvantagem do uso de raios X é no caso de materiais muito densos, como por exemplo estátuas de bronze. Nesses casos, a absorção por esses materiais não permite que eles sejam atravessados pelos raios X, impedindo sua análise. A tomografia de nêutrons pode ser bastante vantajosa nesse caso, superando as limitações dos raios X e complementarmente fornecendo contraste para materiais leves, como resinas, colas, madeiras, etc. Estudos de estátuas de bronze egípcias, por exemplo, permitiram obter informação sobre as técnicas de manufatura, reparos sofridos, e também atestar a autenticidade desses artefatos (de Beer et al. 2009). A principal desvantagem dessa técnica é o fato de ser limitada pelo difícil acesso às fontes de nêutrons disponíveis, como em reatores nucleares.

Uma vez que os dados tenham sido adquiridos e pré-processados para gerar a reconstrução 3D do objeto em estudo, são obtidas imagens que correspondem a "fatias" digitais dos objetos, com valores de intensidade dos pixels relacionados à densidade dos materiais. A partir delas é possível fazer renderizações do volume da amostra, tendo acesso rápido a uma visão geral e tridimensional da morfologia externa e de estruturas internas com elevado contraste. Para análises em maiores detalhes, é possível isolar as estruturas pela técnica de segmentação, a qual consiste em marcar a estrutura de interesse nas diferentes fatias e utilizar essa demarcação para renderizá-la individualmente em 3D. Esse é um processo bastante dispendioso e muitas vezes manual, devido à heterogeneidade das amostras e dos artefatos inerentes à técnica, e depende de um bom treinamento dos usuários e acesso a um poder de computação razoável, bem como softwares específicos, também bastante

5 Reimpresso de Seales B. W. et al. Sci Adv. 2016: 2 (C) Os autores, diretos reservados; licença exlusiva American Association for the Advancement of Science. Distribuido sob Creative Commons Attribution NonCommercial License 4.0 (CC BY-NC) http://creativecommons.org/ licenses/by-nc/4.0/. 
custosos. Atualmente há alguns esforços em criar laboratórios multiusuários de tratamento de dados de tomografia, como no LNLS/CNPEM, mas ainda há falta desse tipo de estrutura diretamente nos museus e institutos de pesquisa em arqueologia, que poderiam ampliar o acesso e popularização da técnica, hoje uma das principais para o estudo de materiais volumétricos, para análises morfológicas, sejam qualitativas ou quantitativas.

\section{Fluorescência de raios $X$}

Talvez uma das técnicas de raios X mais aplicadas em arqueometria (Uda, Demortier, and Nakai 2005), a fluorescência de raios X (XRF, da sigla em inglês para X-Ray Fluorescence) fornece informação sobre a composição elementar do material analisado. Ou seja, é possível identificar e, em alguns casos, quantificar, os elementos químicos presentes mesmo em concentrações muito baixas, como em partes por milhão (ppm) ou até em partes por bilhão (ppb). Seu princípio de funcionamento se baseia no efeito fotoelétrico: Quando o material é irradiado por luz de alta energia (equivalente a comprimento de onda pequeno, como os raios X), elétrons são arrancados das camadas internas dos seus átomos. Esse estado do átomo com elétrons faltantes não é estável, e logo um elétron de uma camada mais externa preenche esse nível desocupado. Nesse processo, ele perde energia emitindo radiação, ou fótons. Esses fótons emitidos são característicos de cada elemento químico, e podem ser usados em sua identificação. Além disso, de maneira simplificada, quanto mais átomos de um determinado elemento químico no material, mais fótons serão produzidos. Com uma calibração adequada, é possível então transformar o número de fótons emitidos na concentração do elemento na amostra, de forma absoluta. A técnica é similar ao EDS (Energy Dispersive Spectroscopy), usada acoplada a Microscópios Eletrônicos de Varredura (MEV), e PIXE (Particle Induced X-ray Emmision), cada uma com diferentes características e escalas de medida. Usualmente MEV/EDS podem ser aplicados em escalas milimétricas (devido à resolução nanometrica da técnica) e PIXE em escalas de dezenas de centímetros, devido à escala sub-milimétrica do feixe. XRF está em uma escala intermediária, mas hoje já existem sistemas que cobrem de nanômetros até dezenas de centímetros, sendo, portanto, extremamente flexível.

Esse tipo de análise pode ser feita de maneira pontual, ou seja, em regiões selecionadas da amostra, que podem ser usadas para estudar em detalhe a composição elementar de uma região específica, ou pode ser usada para mapear os elementos com resolução espacial muito alta, em uma área grande. Tal técnica já foi usada para mapear, por exemplo, pinturas históricas e pré-históricas ocultas (Dik et al. 2008; Reiche and Chalmin 2014). A técnica de micro e nano-fluorescência de raios X usa sistemas especiais para focalizar o feixe de radiação em uma microssonda muito pequena, tipicamente de dezenas de nanômetros até dezenas de micrômetros. Esse sistema pode então ser acoplado com um mecanismo de movimentação motorizado da amostra, permitindo a coleta de informação de muitos pontos sobre uma área extensa (mapeamento 2D) ou mesmo de um volume (tomografia por XRF). A análise de dados é feita por programas específicos, que transformam a informação elementar de cada ponto em um mapa com escala de cor artificial proporcional à concentração. Os mapas químicos assim produzidos podem evidenciar estruturas invisíveis a olho nu, como em pinturas ou diferentes objetos arqueológicos ou históricos (Figura 4).

Para realizar medidas de áreas extensas (centimétricas) em um tempo razoável (de minutos a horas), é necessária uma fonte muito intensa de radiação. Apesar de existirem equipamentos de laboratório que façam mapas por XRF, até mesmo portáteis, grande parte desse tipo de medidas é feita em linhas de luz especializadas em fontes de luz síncrotron. No Brasil, o LNLS/CNPEM conta com uma linha dedicada à fluorescência de raios X (linha XRF), com a flexibilidade de trabalhar com amostras sólidas e líquidas de diferentes naturezas, e atingindo, atualmente, resolução de 25 por $12 \mu$ m (gerando imagens químicas com esse tamanho efetivo de pixel). 


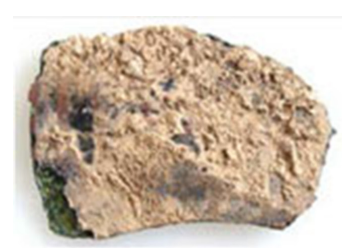

Luz incidente

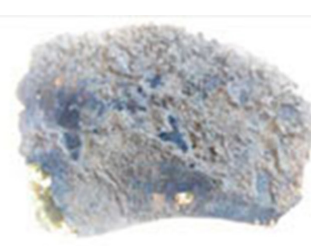

Luz transmitida

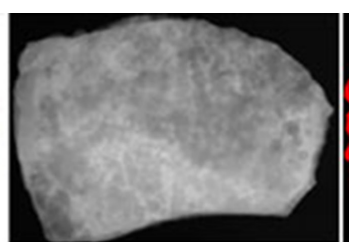

Radiografia

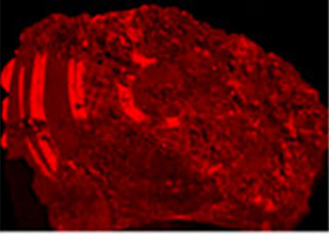

Mapa XRF (ferro)

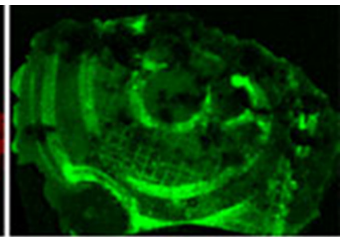

Mapa XRF (chumbo)

Figura 4: Comparação entre imagens óticas e mapeamentos por fluorescência de raios $\mathrm{X}$ dos elementos ferro ( $\mathrm{Fe})$ e chumbo (Pb), mostrando uma pintura invisível a olho nu em um vidro medieval da coleção da Historic England. Adaptado de http://www.blue-scientific.com/analysing-medievalwindow-glass-micro-xrf/.

\section{Espectroscopia de absorção de raios $X$}

A técnica de espectroscopia de raios X (ou XAS, no inglês X-ray Absorption Spectroscopy) baseia-se no estudo do fenômeno da interação de radiação de energia bem definida, dita monocromática, com a matéria. Essa interação é dependente de diversas propriedades do material em estudo, como sua composição elementar, concentração, estado de oxidação, vizinhança química (ou seja, a quem eles estão ligados ou complexados) e mesmo estrutura cristalina. É uma técnica muito poderosa de análise, pois permite medir de forma qualitativa e quantitativa essas propriedades. O resultado produzido pela medida é chamado de espectro, e é um gráfico de uma propriedade da interação (absorção, emissão, reflexão) em função da energia da radiação. As diferentes propriedades do material se refletem na forma desses espectros, e podem ser estudadas de forma ab initio, quando os fenômenos físicos são modelados a partir de princípios fundamentais de maneira a explicar o resultado experimental, ou de forma semi-empírica, comparando os espectros desconhecidos com os de padrões já bem estudados. A última forma, também chamada de método de fingerprinting, permite não apenas identificar materiais puros, mas também misturas complexas, onde é possível recuperar a proporção entre diferentes fases. Essa técnica realizada em síncrotron permite selecionar a energia adequada para estudar especificamente diferentes elementos de interesse arqueológico (por exemplo, ferro, zinco, manganês, cálcio, entre muitos outros), e retirar a informação de seu estado químico (Janssens and Spectus 2010; Chalmin et al. 2007; Chalmin, Farges, and Brown 2009). Por exemplo, para o ferro é possível identificar facilmente seu estado de oxidação ( 0 , para ferro metálico, +2 ou +3 para óxidos, hidróxidos e sais de ferro; por exemplo, o mineral pirita possui Fe ${ }^{2+}$, a hematita é composta por $\mathrm{Fe}^{3+}$ e a magnetita é composta tanto por $\mathrm{Fe}^{2+}$ quanto $\mathrm{Fe}^{3+}$ ) e mesmo diretamente em qual mineral ele está, ou quais fases estão presentes, se for uma mistura. Cada um deles possui uma assinatura espectral bem característica e facilmente separável. Mesmo materiais com a mesma valência, como os pigmentos vermelhos feitos de hematita e maghemita (ambos $\mathrm{Fe}_{2} \mathrm{O}_{3}$, portanto $\mathrm{Fe}^{3+}$ ) podem ser distinguidos aplicando a técnica de XAS (Figura 5).

Essa técnica é similar à espectroscopia Mössbauer, amplamente usada em arqueometria, especialmente por sua portabilidade, porém com a vantagem de ser facilmente aplicável a vários elementos além do ferro, com medidas mais rápidas e de melhor sensibilidade para diferentes fases (Wagner and Kyek 2004). Além disso, atualmente é possível aplicar a técnica de espectroscopia de raios X em síncrotrons em micro e nano escala, obtendo informação química refinada com resolução espacial, ampliando o conhecimento obtido com o mapeamento elementar por XRF. No LNLS/CNPEM, as linhas SXS, XAFS2 e XRF são capazes de fazer a caracterização espectroscópica de elementos com números atômicos a partir do alumínio (ou seja, incluindo alumínio e elementos mais pesados: alumínio, silício, fósforo, enxofre, cloro e assim por diante), com resolução micrométrica. 


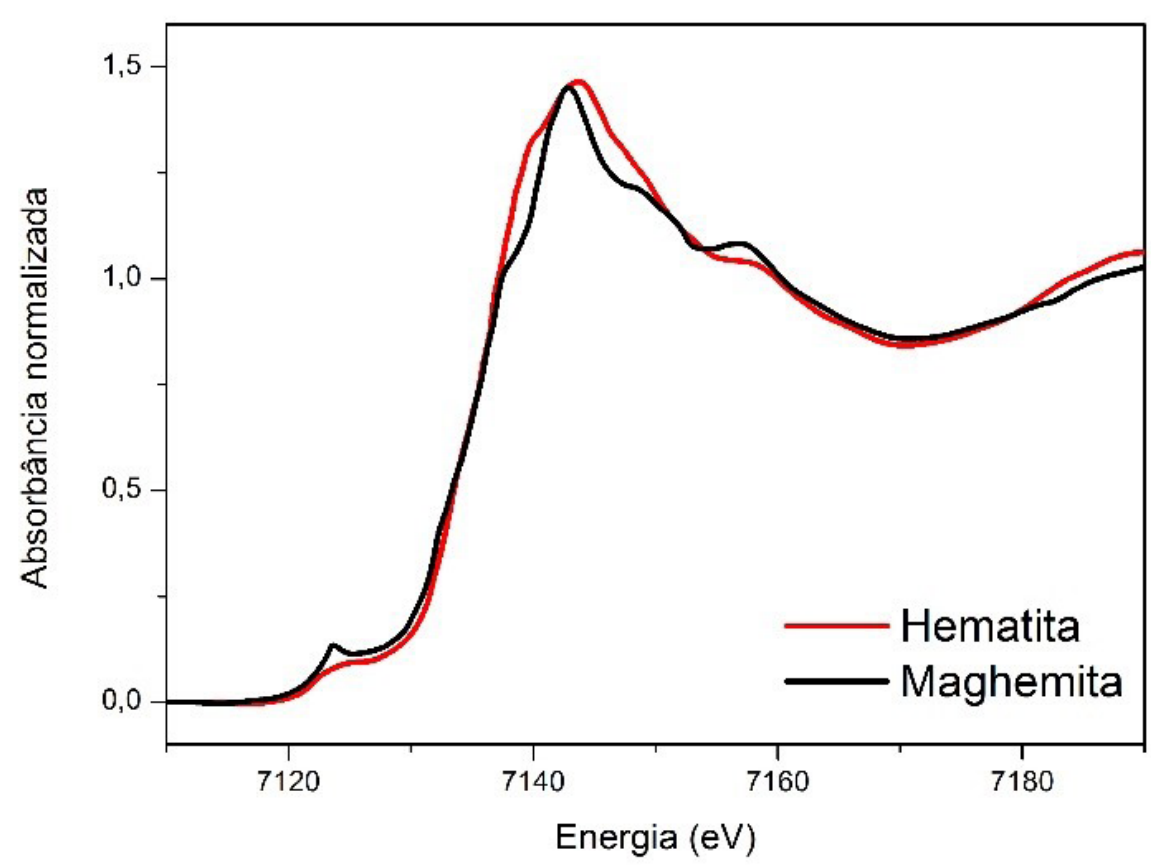

Figura 5: Comparação entre o espectro de absorbância de raios X dos minerais hematita (linha vermelha) e magnetita (linha preta), ambos usados como vermelhos em pinturas pré-históricas, como os vermelhos de Altamira, Espanha (hematita) e de Provence, França (magnetita).

O uso da técnica de fingerprinting em arqueometria e no estudo de materiais naturais está ganhando aceitação, porém ainda é necessária a produção de bancos de dados específicos, com materiais reais e, em especial, materiais do patrimônio brasileiro. Isso é importante pois o espectro pode ser alterado dependendo da forma de preparo do material, da matrizsuporte e de outros fatores. Uma interpretação direta e ingênua dos resultados pode ser enganosa, e muitas vezes difícil de ser percebida se não houver uma boa interação entre o especialista da técnica e o arqueólogo, trazendo o contexto do material.

No Brasil, a arqueometria é uma técnica ainda em franca expansão. Suas aplicações são principalmente baseadas no uso de equipamentos portáteis, como microfluorescência de raios X e microespectroscopia Raman, mas também já estão sendo usadas técnicas analíticas quantitativas laboratoriais, como espectroscopia de infravermelho por transformada de Fourier e microtomografia computadorizada (Alves et al. 2014; Appoloni 2013; Appoloni, Parreira, and Lopes 2008; Puglieri et al. 2019; Ribeiro et al. 2015; Santos et al. 2009; Silva et al. 2004).

\section{UM FUTURO BRILHANTE PARA O BRASIL: A NOVA FONTE DE LUZ SÍNCROTRON SIRIUS}

O LNLS/CNPEM está atualmente construindo uma nova fonte de luz síncrotron, denominada Sirius, assim batizada devido à estrela mais brilhante do firmamento ${ }^{6}$. Esse novo grande laboratório de pesquisa é um dos maiores projetos de pesquisa do país, sendo financiado primariamente pelo MCTIC, e está sendo construído no campus do CNPEM em Campinas/ SP (Figura 6). 

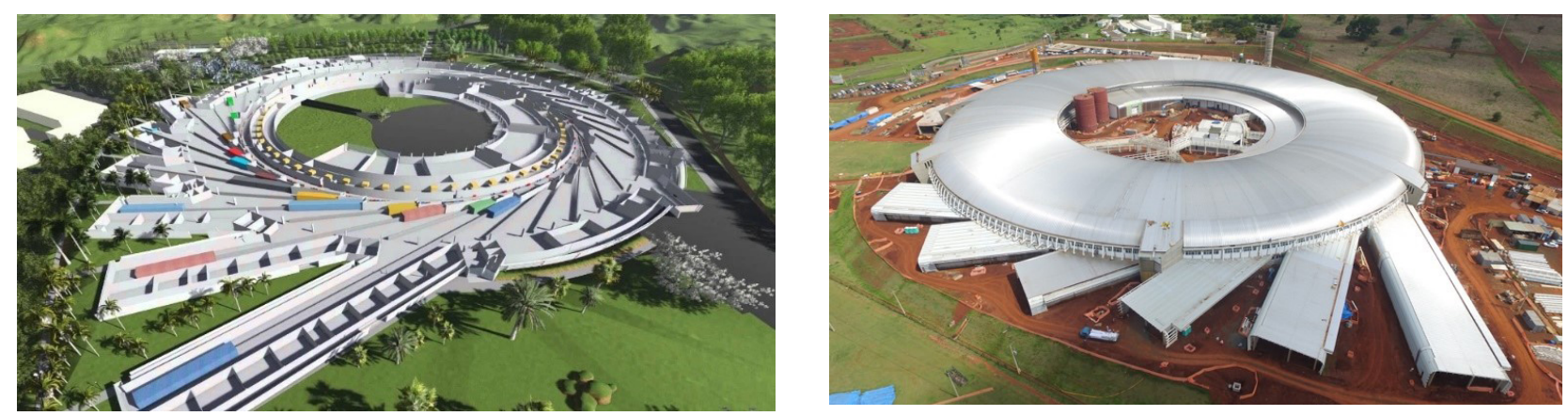

Figura 6: (esquerda) Concepção artística da nova fonte de luz síncrotron Sirius e (direita) estado das obras em março de 2018. Fonte: LNLS/

$$
\text { Divulgação. }
$$

O novo acelerador é um dos projetos mais ambiciosos do mundo em fontes síncrotron, e está sendo denominado como uma fonte de $4^{\mathrm{a}}$ geração, superior aos atuais síncrotrons de $3^{\mathrm{a}}$ geração existentes. É um projeto extremamente competitivo, e irá permitir avançar as pesquisas com materiais dos mais diversos tipos, incluindo os de interesse arqueológico. Dentre as vantagens em relação ao acelerador brasileiro atual estão: maior sensibilidade a elementos pesados, estudo de fases ainda mais diluídas, tempos de medidas muito mais rápidos, capacidade de trabalhar com amostras maiores e altíssima resolução espacial, atingindo poucos nanômetros. O Sirius será um dos mais potentes microscópios de raios X do mundo, sendo capaz de mapear e caracterizar amostras com as mais diferentes técnicas, desde a macro até a nano-escala.

O Sirius irá manter e ampliar a missão do LNLS de ser um laboratório de pesquisa aberto a usuários externos de diferentes comunidades de pesquisa, e abrirá portas para estudos antes impossíveis na área de arqueometria, não apenas no Brasil, como no mundo. 


\section{REFERÊNCIAS}

ALVES, Felipe; Estanislau João, Gleyko Madson, Ceciany Soarigues, Igor Ricart, Marcos Andrade, Viviane Khoury, and Paula Frassinetti. 2014. "Raios x Aplicados à Arqueometria." Cadernos de Graduação - Ciências Biológicas e Saúde 1 (3): 59-68.

APPOLONI, Carlos Roberto. 2013. "Recent Developments in Atomic/Nuclear Methodologies Used for the Study of Cultural Heritage Objects.” AIP Conference Proceedings 1529 (May): 30-39. https://doi.org/10.1063/1.4804077.

APPOLONI, Carlos Roberto, Paulo S Parreira, and Fabio Lopes. 2008. "Métodos e Técnicas de Caracterização Aplicadas Ao Estudo Do Patrimônio Cultural: Trabalhos Desenvolvidos Pelo LFNA-DFIS.” Revista CPC, no. 6 (October): $198-207$. https://doi.org/10.11606/issn.1980-4466.v0i6p198-207.

BAUM, Daniel, Norbert Lindow, Hans-Christian Hege, Verena Lepper, Tzulia Siopi, Frank Kutz, Kristin Mahlow, and Heinz-Eberhard Mahnke. 2017. "Revealing Hidden Text in Rolled and Folded Papyri.” Applied Physics A 123 (3): 171. https://doi.org/10.1007/s00339-017-0808-6.

BEER, Frikkie.C. de, Hazel. Botha, Ernst. Ferg, R. Grundlingh, and A. Smith. 2009. "Archaeology Benefits from Neutron Tomography Investigations in South Africa." Nuclear Instruments and Methods in Physics Research Section A: Accelerators, Spectrometers, Detectors and Associated Equipment 605 (1-2): 167-70. https://doi.org/10.1016/j. nima.2009.01.213.

BERTRAND, Loïc, Laurianne Robinet, Mathieu Thoury, Koen Janssens, Serge X. Cohen, and Sebastian Schöder. 2012. “Cultural Heritage and Archaeology Materials Studied by Synchrotron Spectroscopy and Imaging." Applied Physics A: Materials Science and Processing 106 (2): 377-96. https://doi.org/10.1007/s00339-011-6686-4.

CHALMIN, E., F. Farges, and G. E. Brown. 2009. “A Pre-Edge Analysis of Mn K-Edge XANES Spectra to Help Determine the Speciation of Manganese in Minerals and Glasses." Contributions to Mineralogy and Petrology 157 (1): 111-26. https://doi.org/10.1007/s00410-008-0323-z.

CHALMin, E., F. Farges, C. Vignaud, J. Susini, M. Menu, and G. E. Brown. 2007. "Discovery of Unusual Minerals in Paleolithic Black Pigments from Lascaux (France) and Ekain (Spain).” In AIP Conference Proceedings, 882:220-22. AIP. https://doi.org/10.1063/1.2644480.

DIK, Joris, Koen Janssens, Geert Van Der Snickt, Luuk Van Der Loeff, Karen Rickers, and Marine Cotte. 2008. "Visualization of a Lost Painting by Vincent van Gogh Using Synchrotron Radiation Based X-Ray Fluorescence Elemental Mapping." Analytical Chemistry 80 (16): 6436-42. https://doi.org/10.1021/ac800965g.

JANSSENS, Koen. 2011. "Synchrotron Radiation in Art and Archaeology,” 883-84. https://doi.org/10.1039/c1ja90015c.

JANSSENS, Koen, and C O N Spectus. 2010. “Spectroscopy for Art Conservation : Looking Back” 43 (6): $705-14$.

MALDANIS, Lara, Murilo Carvalho, Mariana Ramos Almeida, Francisco Idalécio Freitas, José Artur Ferreira Gomes de Andrade, Rafael Silva Nunes, Carlos Eduardo Rochitte, Poppi, Ronei Jesus, Freitas, Raul Oliveira, Rodrigues, Fábio, Siljeström, Sandra, Lima, Frederico Alves, Galante, Douglas, Carvalho, Ismar S, Perez, Carlos Alberto, de Carvalho, Marcelo Rodrigues, Bettini, Jefferson, Fernandez, Vincent, Xavier-Neto, José 2016. "Heart Fossilization Is Possible and Informs the Evolution of Cardiac Outflow Tract in Vertebrates.” ELife 5: 1-12. https://doi.org/10.7554/eLife.14698.

MILLS, David, Oksana Samko, Paul Rosin, Kate Thomas, Tim Wess, and Graham R Davis. 2012. “Apocalypto: Revealing the Unreadable." SPIE Optical Engineering + Applications 8506: 85060A-5. https://doi.org/10.1117/12.928917. 
MOCELLA, Vito, Emmanuel Brun, Claudio Ferrero, and Daniel Delattre. 2014. "Revealing Letters in Rolled Herculaneum Papyri by X-Ray Phase-Contrast Imaging.” Nature Communications 6 (May 2014): 5895. https://doi.org/10.1038/ ncomms6895.

PUGLIERI, Thiago Sevilhano, Rafael Guedes Milheira, Eliane Aparecida Del Lama, Patricia Marques Magon, and Sailer Santos do Santos. 2019. "Multi-Technique Investigation of Potshards of a Cerrito (Earthen Mound) from Southern Brazil." Spectrochimica Acta - Part A: Molecular and Biomolecular Spectroscopy 206 (January): 48-56. https://doi. $\operatorname{org} / 10.1016 /$ j.saa.2018.07.084.

REICHE, Ina, and Emilie Chalmin. 2014. 'De Lascaux à Van Gogh : De 1' Analyse de Prélèvements à 1' Imagerie Chimique Non-Invasive Des Peintures." Chimie et Archéométrie, 1-11.

RIBEIRO, M N, Ana Letícia C De Oliveira, Renato P De Freitas, and Cristiane Calza. 2015. “Análise De Pigmentos Por Fluosrecência De Raios X Em Escultura Barroca Do Século Xviii” 3 (século XVIII).

SANTOS, J. O., C. S. Munita, R. G. Toyota, C. Vergne, R. S. Silva, and P. M.S. Oliveira. 2009. “The Archaeometry Study of the Chemical and Mineral Composition of Pottery from Brazil's Northeast." Journal of Radioanalytical and Nuclear Chemistry 281 (2): 189-92. https://doi.org/10.1007/s10967-009-0119-2.

SEALES, William Brent, Clifford Seth Parker, Michael Segal, Emanuel Tov, Pnina Shor, and Yosef Porath. 2016. "From Damage to Discovery via Virtual Unwrapping: Reading the Scroll from En-Gedi." Science Advances 2 (9): 1-10. https://doi.org/10.1126/sciadv.1601247.

SILVA, Fabíola Andréa, Carlos R. Appoloni, Fernando R. E. Quiñones, Ademilson O. Santos, Luzeli M. da Silva, Paulo F. Barbieri, and Virgilio F. Nascimento Filho. 2004. "A Arqueometria e a Análise de Artefatos Cerâmicos: Um Estudo de Fragmentos Cerâmicos Etnográficos e Arqueológicos Por Fluorescência de Raios X ( EDXRF ) e Transmissão Gama.” Revista de Arqueologia 17: 41-61.

SMITH, Tanya M., Paul Tafforeau, Adeline Le Cabec, Anne Bonnin, Alexandra Houssaye, Joane Pouech, Jacopo MoggiCecchi, Manthi, Fredrick, Ward, Carol, Makaremi, Masrour, Menter, Colin G. 2015. "Dental Ontogeny in Pliocene and Early Pleistocene Hominins.” PLoS ONE 10 (2): 1-20. https://doi.org/10.1371/journal.pone.0118118.

UDA, Masayuki, Guy Demortier, and Izumi Nakai. 2005. X-Rays for Archaeology. Springer.

WAGNER, F E, and A Kyek. 2004. "Mossbauer Spectroscopy in Archaeology: Introduction and Experimental Considerations." Hyperfine Interactions 154 (1-4): 5-33.

Recebido em: 22/05/2018 Aprovado em: 16/08/2018

Publicado em: 30/11/2018 